\title{
CONVOLUTION INTEGRAL EQUATION OF FREDHOLM TYPE WITH CERTAIN TRANCEDENTAL FUNCTIONS
}

\author{
V. B. L. CHAURASIA AND VISHAL SAXENA
}

\begin{abstract}
The aim of this paper is to establish a solution of a certain class of convolution integral equation of Fredholm type whose kernel involve certain product of special function by using Riemann-Liouville and Weyl fractional integral operators. Some interesting particular cases are also considered.
\end{abstract}

\section{Introduction}

The following is the special case of Raizada's generalized polynomial set and defined as:

$$
\begin{aligned}
S_{n}^{\alpha, \beta, 0}[x ; r, q, A, B, k, \ell] & =(A x+B)^{-\alpha} \exp \left(\beta x^{r}\right) T_{k, \ell}^{n}\left[(a x+b)^{\alpha+q n} \exp \left(-\beta x^{r}\right)\right] \\
& =\sum_{e, p, u, v} \phi(e, p, u, v) x^{L},
\end{aligned}
$$

where

$$
\begin{gathered}
\phi(e, p, u, v)=B^{q n-p} \ell^{n} \frac{(-1)^{p}(-v)_{u}(-p)_{e}(\alpha)_{p}}{u ! v ! e ! p !} \frac{(-\alpha-q n)_{e}}{(1-\alpha-p)_{e}}\left(\frac{\alpha+k+r u}{\ell}\right)_{n} A^{p} \beta^{v}, \\
L=\ell n+p+r v, \quad(p, v=0,1, \ldots, n)
\end{gathered}
$$

and

$$
\sum_{e, p, u, v}=\sum_{v=0}^{n} \sum_{u=0}^{v} \sum_{p=0}^{n} \sum_{e=0}^{p}
$$

The polynomial set defined by (1.1) is a very general in nature and it unifies and extends a number of classical polynomials introduced and studied by various research workers such as Chatterjea (1969, 1966), Gould and Hopper (1962), Krall and Frink (1949), Srivastava and Singhal (1971) etc.

We have made an attempt, in the present paper, to derive an exact solution of the following convolution integral equation of Fredholm type

$$
\int_{0}^{\infty} y^{-\mu} u\left(\frac{x}{y}\right) f(y) d y=g(x), \quad(x>0)
$$

Received March 1, 2007; revised August 14, 2007.

2000 Mathematics Subject Classification. 33C99, 26A33, 45B05.

Key words and phrases. Riemann-Liouville fractional integral, Weyl fractional integral, Mellin transform technique, $\bar{H}$-function. 
where $g$ is a prescribed function, $f$ is a unknown function to be determined and the kernel $u(x)$ is given by

$$
\begin{aligned}
& u(x)=S_{n}^{\alpha, \beta, 0}\left[z x^{\rho} ; r, q, A, B, k, \ell\right] H_{P_{1}, Q_{1}}^{M_{1}, N_{1}}\left[\left.\tau\left(x^{\sigma}\right)\right|_{\left(f_{Q}, F_{Q}\right)} ^{\left(e_{p}, E_{P}\right)}\right] \\
& \times \bar{H}_{P, Q}^{M, N}\left[\left.w x^{\lambda}\right|_{\left(b_{j}, \beta_{j}^{\prime}\right)_{1, M},\left(b_{j}, \beta_{j}^{\prime}: B_{j}\right)_{M+1, Q}} ^{\left(a_{j}, \alpha_{\prime}^{\prime}: A_{j}\right)_{1, N},\left(a_{j}, \alpha_{j}^{\prime}\right)_{N+1, P}}\right],
\end{aligned}
$$

also $H_{P_{1}, Q_{1}}^{M_{1}, N_{1}}[z]$ is the well known Fox' H-function (1961).

The $\bar{H}$ function defined by Inayat-Hussain (1987) as:

$$
\bar{H}_{P, Q}^{M, N}[z]=\bar{H}_{P, Q}^{M, N}\left[\left.z\right|_{\left(b_{j}, \beta_{j}^{\prime}\right)_{1, M},\left(b_{j}, \beta_{j}^{\prime}: B_{j}\right)_{M+1, Q}} ^{\left(a_{j}, \alpha_{j}^{\prime}: A_{j}\right)_{1, N} ;\left(a_{j}, \alpha^{\prime}\right)_{N+1, P}}\right]=\frac{1}{2 \pi i} \int_{-i \infty}^{i \infty} \phi(s) z^{s} d s,
$$

where

$$
\phi(s)=\frac{\prod_{j=1}^{M} \Gamma\left(b_{j}-\beta_{j}^{\prime} s\right) \prod_{j=1}^{N}\left\{\Gamma\left(1-a_{j}+\alpha_{j}^{\prime} s\right)\right\}^{A j}}{\prod_{j=M+1}^{Q}\left\{\Gamma\left(1-b_{j}+\beta_{j}^{\prime} s\right)\right\}^{B j} \prod_{j=N+1}^{P} \Gamma\left(a_{j}-\alpha_{j}^{\prime} s\right)}
$$

which contains fractional powers of some of the $\Gamma$-functions. Here $Z$ may be real or complex but is not equal to zero and an empty product is interpreted as unity, $P, Q, M$ and $N$ are integers such that $1 \leq M \leq Q, 0 \leq N \leq P, \alpha_{j}^{\prime}(j=1, \ldots, P), \beta_{j}^{\prime}(j=1, \ldots, Q)$ are complex numbers. The exponents $A_{j}(j=1, \ldots, N)$ and $B_{j}(j=M+1, \ldots, Q)$ can take non-integer values when these exponents take integer values, the $\bar{H}$ function reduces to the familiar $\mathrm{H}$-function due to Fox.

Srivastava (1972) introduced the general class of polynomial

$$
S_{n_{1}}^{m_{1}}[A]=\sum_{k_{1}=0}^{\left[n_{1} / m_{1}\right]} \frac{\left(-n_{1}\right)_{m_{1}} k_{1}}{k_{1} !} A^{\prime k} A_{n_{1}, k_{1}}
$$

where $m_{1}$ is an arbitrary positive integer and the coefficients $A_{n_{1}, k_{1}}\left(n_{1}, k_{1} \geq 0\right)$ are aribitrary constants, real or complex.

Let $J$ denote the space of all functions $f$ which are defined on $R^{+}=[0, \infty]$ and satisfy

(i) $f \in C^{\infty}\left(R^{+}\right)$

(ii) $\lim _{x \rightarrow \infty}\left[x^{k} f^{r}(x)\right]=0$ for all non-negative integers $k$ and $r$, and

(iii) $f(x)=0(1)$ as $x \rightarrow 0$

$J$ corresponds to the space of good functions defined on the whole real line (Miller 1975).

For the present study, we shall also require the Riemann-Liouville fractional integral (of order $\mu$ ) defined by

$$
D^{-\mu}\{f(x)\}={ }_{0} D_{x}^{-\mu}\{f(x)\}
$$




$$
=\frac{1}{\Gamma(\mu)} \int_{0}^{x}(x-w)^{\mu-1} f(w) d w, \quad(\operatorname{Re}(\mu)>0 ; f \in J),
$$

and the Weyl fractional integral (of order $h$ ) defined by

$$
\begin{aligned}
W^{-h_{\{}}\{f(x)\} & ={ }_{x} D_{\infty}^{-h_{1}}\{f(x)\} \\
& =\frac{1}{\Gamma(h)} \int_{x}^{\infty}(\zeta-x)^{h-1} f(\zeta) d \zeta(\operatorname{Re}(h)>0 ; f \in J) .
\end{aligned}
$$

\section{Preliminary Results}

Lemma 1. Assuming the following that

(i) $P, Q, M, N$ are integers such that $1 \leq M \leq Q, 0 \leq N \leq P, \alpha_{j}^{\prime}(j=1, \ldots, P), \beta_{j}^{\prime}(j=1, \ldots, Q)$ are complex numbers;

(ii) $\operatorname{Re}(\mu)>\operatorname{Re}(h) ; \operatorname{Re}\left(h+\rho L+\sigma \eta_{G}+\lambda \frac{b_{j}}{\bar{\beta}_{j}^{\prime}}\right)>0$, where $j=1, \ldots, M, M$ is a positive integer, $\lambda \geq 0,(L=\ell n+p+r v), p, v=0,1, \ldots, n ; w \geq 0, \sigma \geq 0 ;$

(iii) $|\arg w|<\frac{1}{2} \pi \Omega_{2}$, where

$$
\Omega_{2}=\sum_{j=1}^{M}\left|\beta_{j}^{\prime}\right|+\sum_{j=1}^{N}\left|A_{j} \alpha_{j}^{\prime}\right|-\sum_{j=M+1}^{Q}\left|B_{j} \beta_{j}^{\prime}\right|-\sum_{j=N+1}^{P}\left|\alpha_{j}^{\prime}\right|>0 ;
$$

then

$$
\begin{aligned}
W^{h-\mu} & \left\{y^{-\mu} S_{n}^{\alpha, \beta, 0}\left[z\left(\frac{x}{y}\right)^{\prime} ; r, q, A, B, k, \ell\right] H_{P_{1}, Q_{1}}^{M_{1}, N_{1}}\left[\left.\tau\left(\frac{x}{y}\right)^{\sigma}\right|_{\left(f_{Q}, F_{Q}\right)} ^{\left(e_{p}, E_{P}\right)}\right]\right. \\
& \left.\times \bar{H}_{P, Q}^{M, N}\left[w\left(\frac{x}{y}\right)^{\lambda}||_{\left(b_{j}, \beta_{j}^{\prime}\right)_{1, M},\left(b_{j}, \beta_{j}^{\prime}: B_{j}\right)_{M+1, Q}}^{\left(a_{j}, \alpha_{j}^{\prime}: A_{j}\right)_{1, N},\left(a_{j}, \alpha_{j}^{\prime}\right)_{N+1, P}}\right]\right\} \\
= & y^{-h} \sum_{e, p, u, v} \phi_{1}(e, p, u, v) z^{L} \sum_{G=0}^{\infty} \sum_{g=1}^{M_{1}} \frac{(-1)^{G}}{G ! F_{g}} \phi_{2}\left(\eta_{G}\right) \tau^{\eta_{G}}\left(\frac{x}{y}\right)^{\rho L+\sigma \eta_{G}} \\
& \times \bar{H}_{P+1, Q+1}^{M, N+1}\left[\left.w\left(\frac{x}{y}\right)^{\lambda}\right|_{\left(b_{j}, \beta_{j}^{\prime}\right)_{1, M},\left(b_{j}, \beta_{j}^{\prime}: B_{j}\right)_{M+1, Q},\left(1-\mu-\sigma \eta_{G}-\rho L, \lambda ; 1\right)} ^{\left(1-h-\rho-\sigma \eta_{G}, \lambda ; 1\right)\left(a_{j}, \alpha_{j}^{\prime}: A_{j}\right)_{1, N},\left(a_{j}, \alpha_{j}^{\prime}\right)_{N+1, P}}\right] .
\end{aligned}
$$

Proof. Making use of definition (1.11) and the series representations (1.1) and for the generalized polynomial set and the Fox's H-function (see Skibiñski, (1970)) respectively in the left-hand side of (2.1), then expressing the Fox's $\overline{\mathrm{H}}$-function in Mellin-Barnes type contour integral and changing the order of summations and integration (which is justified under the conditions stated), we find that left-hand side of (2.1)

$$
\begin{aligned}
= & \frac{1}{\Gamma(\mu-h) 2 \pi i} \sum_{e, p, u, v} \phi_{1}(e, p, u, v) \sum_{G=0}^{\infty} \sum_{g=1}^{M_{1}} \frac{(-1)^{G}}{G ! F_{g}} \phi_{2}\left(\eta_{G}\right) \tau^{\eta_{G}} z^{L} \int_{-i \infty}^{i \infty} \phi_{3}(s) w^{s} \\
& \times x^{\rho L+\sigma \eta_{G}+\lambda s}\left\{\int_{y}^{\infty}(\xi-y)^{\mu-h-1} \xi^{-\mu-\rho L-\lambda s-\sigma \eta_{G}} d \xi\right\} d s .
\end{aligned}
$$


Now evaluating the inner $\xi$ integral in (2.2) with the help of a known result (Erdélyi et al. 1954, p.201, Eq.(6)) and then reinterpreting the resulting Mellin-Barnes contour integral in terms of $\bar{H}$-function, we arrive at the desires result (2.1).

Lemma 2. Under the conditions stated with Lemma 1, we have

$$
\begin{aligned}
\int_{0}^{\infty} y^{-h} \sum_{e, p, u, v} \phi_{1}(e, p, u, v) z^{L} \sum_{G=0}^{\infty} \sum_{g=1}^{M_{1}} \frac{(-1)^{G}}{G ! F_{g}} \phi_{2}\left(\eta_{G}\right) \tau^{\eta_{G}}\left(\frac{x}{y}\right)^{\rho L+\sigma \eta_{G}} \\
\quad \times \bar{H}_{P+1, Q+1}^{M, N+1}\left[\left.w\left(\frac{x}{y}\right)^{\lambda}\right|_{\left(b_{j}, \beta_{j}^{\prime}\right)_{1, M},\left(b_{j}, \beta_{j}^{\prime}: B_{j}\right)_{M+1, Q},\left(1-\mu-\sigma \eta_{G}-\rho L, \lambda ; 1\right)} ^{\left(1-h L-\sigma \eta_{G}, \lambda ; 1\right)\left(a_{j}, \alpha_{j}^{\prime}: A_{j}\right)_{1, N},\left(a_{j}, \alpha_{j}^{\prime}\right)_{N+1, P}}\right] f(y) d y \\
=\int_{0}^{\infty} \zeta^{-\mu} S_{n}^{\alpha, \beta, 0}\left[z\left(\frac{x}{\zeta}\right)^{\rho} ; r, q, A, B, k, \ell\right] H_{P_{1}, Q_{1}}^{M_{1}, N_{1}}\left[\left.\tau\left(\frac{x}{\zeta}\right)^{\sigma}\right|_{\left(f_{Q}, F_{Q}\right)} ^{\left(e_{p}, E_{P}\right)}\right] \\
\quad \times \bar{H}_{P, Q}^{M, N}\left[\left.w\left(\frac{x}{\zeta}\right)^{\lambda}\right|_{\left(b_{j}, \beta_{j}^{\prime}\right)_{1, M},\left(b_{j}, \beta_{j}^{\prime}: B_{j}\right)_{M+1, Q}} ^{\left(a_{j}, \alpha_{j}^{\prime}: A_{j}\right)_{1, N},\left(a_{j}, \alpha_{j}^{\prime}\right)_{N+1, P}}\right] D^{\left.h-\mu_{\{f} f(\zeta)\right\} d \zeta,}
\end{aligned}
$$

provided $f \in J$ and $x>0$.

Proof. Let $\Delta$ denote the left hand side of (2.3). Then using Lemma 1 and applying (1.11), we get

$$
\begin{aligned}
\Delta= & \int_{0}^{\infty}\left\{\frac{1}{\Gamma(\mu-h)} \int_{y}^{\infty}(\zeta-y)^{\mu-h-1} \zeta^{-\mu} S_{n}^{\alpha, \beta, 0}\left[z\left(\frac{x}{\zeta}\right)^{\rho} ; r, q, A, B, k, \ell\right]\right. \\
& \left.\times H_{P_{1}, Q_{1}}^{M_{1}, N_{1}}\left[\left.\tau\left(\frac{x}{\zeta}\right)^{\sigma}\right|_{\left(f_{Q}, F_{Q}\right)} ^{\left(e_{p}, E_{P}\right)}\right] \bar{H}_{P, Q}^{M, N}\left[w\left(\frac{x}{\zeta}\right)^{\lambda} \mid \begin{array}{l}
\left(a_{j}, \alpha_{j}^{\prime}: A_{j}\right)_{1, N},\left(a_{j}, \alpha_{j}^{\prime}\right)_{N+1, P} \\
\left(b_{j}, \beta_{j}^{\prime}\right)_{1, M},\left(b_{j}, \beta_{j}^{\prime}: B_{j}\right)_{M+1, Q}
\end{array}\right] d \zeta\right\} d(y) d y \\
= & \int_{0}^{\infty} \zeta^{-\mu} S_{n}^{\alpha, \beta, 0}\left[z\left(\frac{x}{\zeta}\right)^{\rho} ; r, q, A, B, k, \ell\right] H_{P_{1}, Q_{1}}^{M_{1}, N_{1}}\left[\left.\tau\left(\frac{x}{\zeta}\right)^{\sigma}\right|_{\left(f_{Q}, F_{Q}\right)} ^{\left(e_{p}, E_{P}\right)}\right] \\
& \times \bar{H}_{P, Q}^{M, N}\left[w\left(\frac{x}{\zeta}\right)^{\lambda}||_{\left(b_{j}, \beta_{j}^{\prime}\right)_{1, M},\left(b_{j}, \beta_{j}^{\prime}: B_{j}\right)_{M+1, Q}}^{\left(a_{j}, \alpha_{j}^{\prime}: A_{j}\right)_{1, N},\left(a_{j}, \alpha_{j}^{\prime}\right)_{N+1, P}}\right]\left\{\int_{y}^{\infty} \frac{(\zeta-y)^{\mu-h-1}}{\Gamma(\mu-h)} f(y) d y\right\} d \zeta,
\end{aligned}
$$

where it has been assumed that the change of order of integration is permissible as in the proof of Lemma 1. Now by using (1.10), we get easily the right hand side of (2.3).

\section{Main Theorem}

If $f \in J, D^{\mu-h_{\{}}\{\}$exists, $\lambda>0, x>0,|\arg w|<\frac{\pi}{2} \Omega_{2}, \Omega_{2}>0, \operatorname{Re}(\mu)>\operatorname{Re}(h)>0$, then the solution of the integral equation (1.5) is given by

$$
f(x)=\frac{\lambda}{2 \pi i} x^{\mu-1} \lim _{\gamma \rightarrow \infty} \int_{c-i \gamma}^{c+i \gamma} \frac{x^{-s} \phi(s)}{\psi(s, z, \tau, w)} d s,
$$

where

$$
\phi(s)=\int_{0}^{\infty} x^{s-1} g(x) d x
$$

and

$$
\psi(s, z, \tau, w)=\sum_{e, p, u, v} \phi_{1}(e, p, u, v) z^{L} \sum_{G=0}^{\infty} \sum_{g=1}^{M_{1}} \frac{(-1)^{G}}{G ! F_{g}} \phi_{2}\left(\eta_{G}\right) \tau^{\eta_{G}}
$$




$$
\times \phi_{3}\left(\frac{-\rho L-s-\sigma \eta_{G}}{\lambda}\right) w^{-\left(\frac{s+\rho L+\sigma \eta_{G}}{\lambda}\right)},
$$

provided that

$$
-\min _{1 \leq j \leq M} \operatorname{Re}\left(b_{j} / B_{j}\right)<\operatorname{Re}\left(\frac{s+\rho L+\sigma \eta_{G}}{\lambda}\right)<\min _{1 \leq j \leq N} \operatorname{Re}\left\{\left(1-a_{j}\right) / A_{j}\right\}
$$

where $L=\ell n+p+r v: p, v=0,1, \ldots, n$.

Proof. Replace $f$ by $\left.D^{\mu-h_{\{}} f\right\}$ in (2.3), we have

$$
\begin{aligned}
g(x)= & \int_{0}^{\infty} y^{-h} \sum_{e, p, u, v} \phi_{1}(e, p, u, v) z^{L} \sum_{G=0}^{\infty} \sum_{g=1}^{M_{1}} \frac{(-1)^{G}}{G ! F_{g}} \phi_{2}\left(\eta_{G}\right) \tau^{\eta_{G}}\left(\frac{x}{y}\right)^{\rho L+\sigma \eta_{G}} \\
& \times \bar{H}_{P+1, Q+1}^{M, N+1}\left[\left.w\left(\frac{x}{y}\right)^{\lambda}\right|_{\left(b_{j}, \beta_{j}^{\prime}\right)_{1, M},\left(b_{j}, \beta_{j}^{\prime}: B_{j}\right)_{M+1, Q},\left(1-\mu-\sigma \eta_{G}-\rho L, \lambda ; 1\right)} ^{\left(1-h-\rho L-\sigma \eta_{G}, \lambda ; 1\right),\left(a_{j}, \alpha_{j}^{\prime}: A_{j}\right)_{1, N},\left(a_{j}, \alpha_{j}^{\prime}\right)_{N+1, P}}\right] \\
& \times D^{\left.\mu-h_{\{f}(y)\right\} d y .}
\end{aligned}
$$

Now taking Mellin transform of both the sides, we get

$$
\begin{aligned}
\phi(s)= & \sum_{e, p, u, v} \phi_{1}(e, p, u, v) z^{L} \sum_{G=0}^{\infty} \sum_{g=1}^{M_{1}} \frac{(-1)^{G}}{G ! F_{g}} \phi_{2}\left(\eta_{G}\right) \tau^{\eta_{G}} y^{-h-\rho L-\eta_{G}}\left\{\int_{0}^{\infty} x^{s+\rho L+\sigma \eta_{G}-1}\right. \\
& \left.\times \bar{H}_{P+1, Q+1}^{M, N+1}\left[\left.w\left(\frac{x}{y}\right)^{\lambda}\right|_{\left(b_{j}, \beta_{j}^{\prime}\right)_{1, M},\left(b_{j}, \beta_{j}^{\prime}: B_{j}\right)_{M+1, Q},\left(1-\mu-\sigma \eta_{G}-\rho L, \lambda ; 1\right)} ^{\left(1-h-\rho L-\sigma \eta_{G}, \lambda ; 1\right),\left(a_{j}, \alpha_{j}^{\prime}: A_{j}\right)_{1, N},\left(a_{j}, \alpha_{j}^{\prime}\right)_{N+1, P}}\right] d x\right\} \\
& \times D^{\left.\mu-h_{\{f}(y)\right\} d y,}
\end{aligned}
$$

where it has been assumed that the change of order of integration is permissible under the conditions stated with the theorem. On evaluating the inner integral, (2.10) reduces to

$$
\frac{\lambda \Gamma(\mu-s) \phi(s)}{\Gamma(h-s) \psi(s, z, \tau, w)}=M\left\{y^{1-h} D^{\mu-h}\{f(y)\} ; s\right\}
$$

which on applying Mellin inversion theorem gives

$$
D^{\mu-h}[f(y)]=\frac{\lambda}{2 \pi i} \lim _{\gamma \rightarrow \infty} \int_{c-i \gamma}^{c+i \gamma} y^{h-s-1} \frac{\Gamma(\mu-s) \phi(x) d s}{\Gamma(h-s) \Psi(s, z, \tau, w)} .
$$

Now operating upon both the sides by $D^{h-\mu}$ (defined by (1.10)) and then on changing the order of integration which is permissible under the conditions stated with the theorem, we obtain

$$
\begin{aligned}
f(y)= & \frac{\lambda}{\Gamma(\mu-h) 2 \pi i} \lim _{\gamma \rightarrow \infty} \int_{c-i \gamma}^{c+i \gamma} \frac{\Gamma(\mu-s)}{\Gamma(h-s) \psi(s, z, \tau, w)} \\
& \times\left\{\int_{0}^{y} \zeta^{h-s-1}(y-\zeta)^{\mu-h-1} d \zeta\right\} \phi(s) d s
\end{aligned}
$$


which on evaluating the inner integral by appealing to the well known definition of Beta function, finally yield the required result (2.6).

Lemma 3. If

(i) $P, Q, M, N$ are integers such that $1 \leq M \leq Q, 0 \leq N \leq P, \alpha_{j}^{\prime}(j=1, \ldots, P), \beta_{j}^{\prime}(j=1, \ldots, Q)$ are complex numbers;

(ii) $\operatorname{Re}(\mu)>\operatorname{Re}(h)$;

$$
\operatorname{Re}\left(h+\rho L+\sigma \eta_{G}+\xi_{k_{1}}+\lambda \frac{b_{j}}{\beta_{j}^{\prime}}\right)>0,
$$

where $j=1, \ldots, M, M$ is an positive integer, $\sigma \geq 0, \lambda \geq 0,(L=\ell n+p+r v),(p, v=$ $0,1, \ldots, n)$;

(iii) $m_{1}$ be an arbitrary positive integer and the coefficients $A_{n_{1}, k_{1}}\left(n_{1}, k_{1} \geq 0\right)$ be arbitrary constants, real or complex;

(iv) $|\arg w|<\frac{1}{2} \pi \Omega_{2}$ where $\Omega_{2}$ is defined by

$$
\Omega_{2}=\sum_{j=1}^{M}\left|B_{j}^{\prime}\right|+\sum_{j=1}^{N}\left|A_{j} \alpha_{j}^{\prime}\right|-\sum_{j=M+1}^{Q}\left|B_{j} B_{j}^{\prime}\right|-\sum_{j=N+1}^{P}\left|\alpha_{j}^{\prime}\right|>0 .
$$

Then

$$
\begin{aligned}
W^{h-\mu} & \left\{y^{-\mu} S_{n}^{\alpha, \beta, 0}\left[z\left(\frac{x}{y}\right)^{\rho} ; r, q, A, B, k, \ell\right] S_{n_{1}}^{m_{1}}\left[A^{\prime}\left(\frac{x}{y}\right)^{\xi}\right]\right. \\
& \left.\times H_{P_{1}, Q_{1}}^{M_{1}, N_{1}}\left[\left.\tau\left(\frac{x}{y}\right)^{\sigma}\right|_{\left(f_{Q}, F_{Q}\right)} ^{\left(e_{p}, E_{P}\right)}\right] \bar{H}_{P, Q}^{M, N}\left[w\left(\frac{x}{y}\right)^{\lambda} \mid \begin{array}{l}
\left(a_{j}, \alpha_{j}^{\prime}: A_{j}\right)_{1, N},\left(a_{j}, \alpha_{j}^{\prime}\right)_{N+1, P} \\
\left(b_{j}, \beta_{j}^{\prime}\right)_{1, M},\left(b_{j}, \beta_{j}^{\prime}: B_{j}\right)_{M+1, Q}
\end{array}\right]\right\} \\
= & y^{-h} \sum_{e, p, u, v} \phi_{1}(e, p, u, v) z^{L} \sum_{k_{1}=0}^{\left[n_{1} / m_{1}\right]} \frac{\left(-n_{1}\right)_{m_{1} k_{1}}}{k !} A^{\prime \xi} A_{n_{1}, k_{1}} \\
& \times \sum_{G=0}^{\infty} \sum_{g=1}^{M_{1}} \frac{(-1)^{G}}{G ! F_{g}} \phi_{2}\left(\eta_{G}\right) \tau^{\eta_{G}}\left(\frac{x}{y}\right)^{\rho L+\sigma \eta_{G}+\xi k_{1}} \\
& \times \bar{H}_{P+1, Q+1}^{M, N+1}\left[\left.w\left(\frac{x}{y}\right)^{\lambda}\right|_{\left(b_{j}, \beta_{j}^{\prime}\right)_{1, M},\left(b_{j}, \beta_{j}^{\prime}: B_{j}\right)_{M+1, Q},\left(1-\mu-\rho L-\sigma \eta_{G}-\xi k_{1}, \lambda ; 1\right)} ^{\left(1-h L-\sigma \eta_{G}-\xi k_{1}, \lambda ; 1\right)\left(a_{j}, \alpha_{j}^{\prime}: A_{j}\right)_{1, N},\left(a_{j}, \alpha_{j}^{\prime}\right)_{N+1, P}}\right] .
\end{aligned}
$$

Proof. To prove Lemma 3, firstly we use the definition of Weyl fractional integral given in (1.11), express the generalized polynomial set, $H$-function in series representation, a general class of polynomials and $\bar{H}$-function, then we change the order of summations and integrations (which is justified under the stated conditions), evaluate the $t$-integral and reinterpreting the resulting Mellin-Barnes contour integral in terms of the $\bar{H}$-function, we get the desired result.

\section{Special cases}


(1) If we set $A=1, B=q=k=0$ and $\ell=-1$ in (2.1), (2.3), (2.6) and (2.14), we obtain the following

$$
\begin{aligned}
& \text { (1.a) } W^{h-\mu}\left\{y^{-\mu} H_{n}^{(r)}\left[z\left(\frac{x}{y}\right)^{\rho}, \alpha, \beta\right] H_{P_{1}, Q_{1}}^{M_{1}, N_{1}}\left[\left.\tau\left(\frac{x}{y}\right)^{\sigma}\right|_{\left(f_{Q}, F_{Q}\right)} ^{\left(e_{p}, E_{P}\right)}\right]\right. \\
& \left.\times \bar{H}_{P, Q}^{M, N}\left[\left.w\left(\frac{x}{y}\right)^{\lambda}\right|_{\left(b_{j}, \beta_{j}^{\prime}\right)_{1, M},\left(b_{j}, \beta_{j}^{\prime}: B_{j}\right)_{M+1, Q}} ^{\left(a_{j}, \alpha_{j}^{\prime}: A_{j}\right)_{1, N}\left(a_{j}, \alpha_{j}^{\prime}\right)_{N+1, P}}\right]\right\} \\
& =y^{-h} \sum_{\nu=0}^{n} \sum_{u=0}^{v} \frac{(-v)_{u}(-\alpha-r u)_{n}}{u ! v !} \beta^{v} z^{r \nu-n} \\
& \times \sum_{G=0}^{\infty} \sum_{g=1}^{M_{1}} \frac{(-1)^{G}}{G ! F_{g}} \phi_{2}\left(\eta_{G}\right) \tau^{\eta_{G}}\left(\frac{x}{y}\right)^{\sigma \eta_{G}+\rho(r v-n)} \\
& \times \bar{H}_{P+1, Q+1}^{M, N+1}\left[w\left(\frac{x}{y}\right)^{\lambda} \mid \begin{array}{l}
\left(1-h-\sigma \eta_{G}-\rho r v+\rho n, \lambda ; 1\right),\left(a_{j}, \alpha_{j}^{\prime} ; A_{j}\right)_{1, N},\left(a_{j}, \alpha_{j}^{\prime}\right)_{N+1, P} \\
\left(b_{j}, \beta_{j}^{\prime}\right)_{1, M},\left(b_{j}, \beta_{j}^{\prime} ; B_{j}\right)_{M+1, Q},\left(1-\mu-\sigma \eta_{G}-\rho r v+\rho n, \lambda ; 1\right)
\end{array}\right] . \\
& \text { (1.b) } \int_{0}^{\infty} \zeta^{-\mu} H_{n}^{(r)}\left[z\left(\frac{x}{\zeta}\right)^{\rho}, \alpha, \beta\right] H_{P_{1}, Q_{1}}^{M_{1}, N_{1}}\left[\left.\tau\left(\frac{x}{y}\right)^{\sigma}\right|_{\left(f_{Q}, F_{Q}\right)} ^{\left(e_{p}, E_{P}\right)}\right] \\
& \times \bar{H}_{P, Q}^{M, N}\left[w\left(\frac{x}{\zeta}\right)^{\lambda} \mid \begin{array}{l}
\left(a_{j}, \alpha_{j}^{\prime}: A_{j}\right)_{1, N},\left(a_{j}, \alpha_{j}^{\prime}\right)_{N+1, P} \\
\left(b_{j}, \beta_{j}^{\prime}\right)_{1, M},\left(b_{j}, \beta_{j}^{\prime}: B_{j}\right)_{M+1, Q}
\end{array}\right] D^{\left.h-\mu_{\{f}(\zeta)\right\} d \zeta} \\
& =\int_{0}^{\infty} y^{-h} \sum_{\nu=0}^{n} \sum_{u=0}^{\nu} \frac{(-v)_{u}(-\alpha-r u)_{n}}{u ! v !} \beta^{v} z^{r v-n} \\
& \times \sum_{G=0}^{\infty} \sum_{g=1}^{M_{1}} \frac{(-1)^{G}}{G ! F_{g}} \phi_{2}\left(\eta_{G}\right) \tau^{\eta_{G}}\left(\frac{x}{y}\right)^{\sigma \eta_{G}+\rho(r v-n)} \\
& \times \bar{H}_{P+1, Q+1}^{M, N+1}\left[\left.w\left(\frac{x}{y}\right)^{\lambda}\right|_{\left(b_{j}, \beta_{j}^{\prime}\right)_{1, M},\left(b_{j}, \beta_{j}^{\prime}: B_{j}\right)_{M+1, Q},\left(1-\mu-\sigma \eta_{G}-\rho r v+\rho n, \lambda ; 1\right)} ^{\left(1-h-\sigma \eta_{G}-\rho r v+\rho n, \lambda ; 1\right),\left(a_{j}, \alpha_{j}^{\prime}: A_{j}\right)_{1, N},\left(a_{j}, \alpha_{j}^{\prime}\right)_{N+1, P}}\right] f(y) d y . \\
& \text { (1.c) } \int_{0}^{\infty} y^{-\mu} H_{n}^{(r)}\left[z\left(\frac{x}{\zeta}\right)^{\rho}, \alpha, \beta\right] H_{P_{1}, Q_{1}}^{M_{1}, N_{1}}\left[\left.\tau\left(\frac{x}{y}\right)^{\sigma}\right|_{\left(f_{Q}, F_{Q}\right)} ^{\left(e_{p}, E_{P}\right)}\right] \\
& \times \bar{H}_{P, Q}^{M, N}\left[\left.w\left(\frac{x}{y}\right)^{\lambda}\right|_{\left(b_{j}, \beta_{j}^{\prime}\right)_{1, M},\left(b_{j}, \beta_{j}^{\prime}: B_{j}\right)_{M+1, Q}} ^{\left(a_{j}, \alpha_{j}^{\prime}: A_{j}\right)_{1, N},\left(a_{j}, \alpha^{\prime}\right)_{N+1, P}}\right] f(y) d y=g(x)
\end{aligned}
$$

has its solution given by

$$
\begin{aligned}
f(x)= & \frac{\lambda x^{\mu-1}}{2 \pi i} \lim _{\gamma \rightarrow \infty} \int_{c-i \gamma}^{c+i \gamma} x^{-s}\left[\sum_{\nu=0}^{n} \sum_{u=0}^{v} \frac{(-v)_{u}(-\alpha-r u)_{n}}{u ! \nu !} \beta^{\nu} z^{r \nu-n} \sum_{G=0}^{\infty} \sum_{g=1}^{M_{1}} \frac{(-1)^{G}}{G ! F_{g}}\right. \\
& \left.\times \phi_{2}\left(\eta_{G}\right) \tau^{\eta_{G}} \phi_{3}\left(\frac{-\rho r \nu+\rho n-s-\sigma \eta_{G}}{\lambda}\right) w^{\left(\frac{-\sigma \eta_{G}-s-\rho r v+\rho n}{\lambda}\right)}\right]^{-1} \phi(s) d s
\end{aligned}
$$

$$
\text { (1.d) } \begin{aligned}
& W^{h-\mu}\left\{y ^ { - \mu } H _ { n } ^ { ( r ) } [ z ( \frac { x } { y } ) ^ { \rho } , \alpha , \beta ] S _ { n _ { 1 } } ^ { m _ { 1 } } [ A ^ { \prime } ( \frac { x } { y } ) ^ { \xi } ] H _ { P _ { 1 } , Q _ { 1 } } ^ { M _ { 1 } , N _ { 1 } } \left[\left.\tau\left(\frac{x}{y}\right)^{\sigma}\right|_{\left(f_{Q}, F_{Q}\right)} ^{\left(e_{p}, E_{P}\right)}\right.\right. \\
& \left.\times \bar{H}_{P, Q}^{M, N}\left[w\left(\frac{x}{y}\right)^{\lambda} \mid \begin{array}{l}
\left(_{j}, \alpha_{j}^{\prime}: A_{j}\right)_{1, N},\left(a_{j}, \alpha_{j}^{\prime}\right)_{N+1, P} \\
\left(b_{j}, \beta_{j}^{\prime}\right)_{1, M},\left(b_{j}, \beta_{j}^{\prime}: B_{j}\right)_{M+1, Q}
\end{array}\right]\right\}
\end{aligned}
$$




$$
\begin{aligned}
= & y^{-h} \sum_{\nu=0}^{n} \sum_{u=0}^{v} \sum_{k_{1}=0}^{\left[n_{1} / m_{1}\right]} \frac{(-v)_{u}(-\alpha-r u)_{n}}{u ! v !} \frac{\left(-n_{1}\right)_{m_{1}, k_{1}}}{k_{1} !} A^{\prime \xi} A_{n_{1}, k_{1}} \beta^{\nu} z^{r v-n} \\
& \times \sum_{G=0}^{\infty} \sum_{g=1}^{M_{1}} \frac{(-1)^{G}}{G ! F_{g}} \phi_{2}\left(\eta_{G}\right) \tau^{\eta_{G}}\left(\frac{x}{y}\right)^{\sigma \eta_{G}+\xi k_{1}+\rho(r v-n)} \\
& \times \bar{H}_{P+1, Q+1}^{M, N+1}\left[w\left(\frac{x}{y}\right)^{\lambda} \mid \begin{array}{l}
\left(1-h-\sigma \eta_{G}-\rho r v+\rho n-\xi k_{1}, \lambda ; 1\right),\left(a_{j}, \alpha_{j}^{\prime}: A_{j}\right)_{1, N},\left(a_{j}, \alpha_{j}^{\prime}\right)_{N+1, P} \\
\left(b_{j}, \beta_{j}^{\prime}\right)_{1, M},\left(b_{j}, \beta_{j}^{\prime}: B_{j}\right)_{M+1, Q},\left(1-\mu-\sigma \eta_{G}-\rho r \nu+\rho n-\xi k_{1}, \lambda ; 1\right)
\end{array}\right] .
\end{aligned}
$$

(2) If we set $n=q=k=B=0, \ell=r=-1$ and $A=1$ in (2.1), (2.3), (2.6) and (2.14), we get the following results

$$
\begin{aligned}
& \text { (2.a) } W^{h-\mu}\left\{y^{-\mu} H_{P_{1}, Q_{1}}^{M_{1}, N_{1}}\left[\left.\tau\left(\frac{x}{y}\right)^{\sigma}\right|_{\left(f_{Q}, F_{Q}\right)} ^{\left(e_{p}, E_{P}\right)}\right]\right. \\
& \left.\times \bar{H}_{P+1, Q+1}^{M, N+1}\left[w\left(\frac{x}{y}\right)^{\lambda} \mid \begin{array}{l}
\left(a_{j}, \alpha_{j}^{\prime}: A_{j}\right)_{1, N},\left(a_{j}, \alpha_{j}^{\prime}\right)_{N+1, P} \\
\left(b_{j}, \beta_{j}^{\prime}\right)_{1, M},\left(b_{j}, \beta_{j}^{\prime}: B_{j}\right)_{M+1, Q}
\end{array}\right]\right\} \\
& =y^{-h} \sum_{G=0}^{\infty} \sum_{g=1}^{M_{1}} \frac{(-1)^{G}}{G ! F_{g}} \phi_{2}\left(\eta_{G}\right) \tau^{\eta_{G}}\left(\frac{x}{y}\right)^{\sigma \eta_{G}} \\
& \times \bar{H}_{P+1, Q+1}^{M, N+1}\left[w\left(\frac{x}{y}\right)^{\lambda} \mid \begin{array}{l}
\left(1-h-\sigma \eta_{G}, \lambda ; 1\right),\left(a_{j}, \alpha_{j}^{\prime}: A_{j}\right)_{1, N},\left(a_{j}, \alpha_{j}^{\prime}\right)_{N+1, P} \\
\left(b_{j}, \beta_{j}^{\prime}\right)_{1, M},\left(b_{j}, \beta_{j}^{\prime}: B_{j}\right)_{M+1, Q},\left(1-\mu-\sigma \eta_{G}, \lambda ; 1\right)
\end{array}\right] . \\
& \text { (2.b) } \int_{0}^{\infty} \zeta^{-\mu} H_{P_{1}, Q_{1}}^{M_{1}, N_{1}}\left[\left.\tau\left(\frac{x}{\zeta}\right)^{\sigma}\right|_{\left(f_{Q}, F_{Q}\right)} ^{\left(e_{p}, E_{P}\right)}\right] \bar{H}_{P, Q}^{M, N}\left[w\left(\frac{x}{\zeta}\right)^{\lambda} \mid\right. \\
& \left.\begin{array}{c}
\left(a_{j}, \alpha_{j}^{\prime}: A_{j}\right)_{1, N},\left(a_{j}, \alpha_{j}^{\prime}\right)_{N+1, P} \\
\left(b_{j}, \beta_{j}^{\prime}\right)_{1, M},\left(b_{j}, \beta_{j}^{\prime}: B_{j}\right)_{M+1, Q}
\end{array}\right] D^{\left.h-\mu_{\{f}(\zeta)\right\} d \zeta} \\
& =\int_{0}^{\infty} y^{-h} \sum_{G=0}^{\infty} \sum_{g=1}^{M_{1}} \frac{(-1)^{G}}{G ! F_{g}} \phi_{2}\left(\eta_{G}\right) \tau^{\eta_{G}}\left(\frac{x}{y}\right)^{\sigma \eta_{G}} \\
& \times H_{P+1, Q+1}^{M, N+1}\left[\left.w\left(\frac{x}{y}\right)^{\lambda}\right|_{\left(b_{j}, \beta_{j}^{\prime}\right)_{1, M},\left(b_{j}, \beta_{j}^{\prime}: B_{j}\right)_{M+1, Q},\left(1-\mu-\sigma \eta_{G}, \lambda ; 1\right)} ^{\left(1-h \eta_{G}, \lambda ; 1\right)\left(a_{j}, \alpha_{j}^{\prime}: A_{j}\right)_{1, N},\left(a_{j}, \alpha_{j}^{\prime}\right)_{N+1, P}}\right] f(y) d y \\
& \text { (2.c) } \int_{0}^{\infty} y^{-\mu} H_{P_{1}, Q_{1}}^{M_{1}, N_{1}}\left[\left.\tau\left(\frac{x}{y}\right)^{\sigma}\right|_{\left(f_{Q}, F_{Q}\right)} ^{\left(e_{p}, E_{P}\right)}\right] \bar{H}_{P, Q}^{M, N}\left[w\left(\frac{x}{y}\right)^{\lambda} \mid\right. \\
& \left.\begin{array}{c}
\left(a_{j}, \alpha_{j}^{\prime}: A_{j}\right)_{1, N},\left(a_{j}, \alpha_{j}^{\prime}\right)_{N+1, P} \\
\left(b_{j}, \beta_{j}^{\prime}\right)_{1, M},\left(b_{j}, \beta_{j}^{\prime}: B_{j}\right)_{M+1, Q}
\end{array}\right] f(y) d y=g(x)
\end{aligned}
$$

has its solution given by

$$
\begin{aligned}
f(x)= & \frac{\lambda x^{\mu-1}}{2 \pi i} \lim _{\gamma \rightarrow \infty} \int_{c-i \gamma}^{c+i \gamma} x^{-s}\left[\sum_{G=0}^{\infty} \sum_{g=1}^{M_{1}} \frac{(-1)^{G}}{G ! F_{g}} \phi_{2}\left(\eta_{G}\right) \tau^{\eta_{G}}\right. \\
& \left.\times \phi_{3}\left(\frac{-s-\sigma \eta_{G}}{\lambda}\right) w^{\left(\frac{-\sigma \eta_{G}-s}{\lambda}\right)}\right]^{-1}
\end{aligned}
$$




$$
\text { (2.d) } \begin{aligned}
& W^{h-\mu}\left\{y^{-\mu} S_{n_{1}}^{m_{1}}\left[A^{\prime}\left(\frac{x}{y}\right)^{\xi}\right] H_{P_{1}, Q_{1}}^{M_{1}, N_{1}}\left[\left.\tau\left(\frac{x}{y}\right)^{\sigma}\right|_{\left(f_{Q}, F_{Q}\right)} ^{\left(e_{p}, E_{P}\right)}\right]\right. \\
& \left.\times \bar{H}_{P, Q}^{M, N}\left[\left.w\left(\frac{x}{y}\right)^{\lambda}\right|_{\left(b_{j}, \beta_{j}^{\prime}\right)_{1, M},\left(b_{j}, \beta_{j}^{\prime}: B_{j}\right)_{M+1, Q}} ^{\left(a_{j}, \alpha_{j}^{\prime}: A_{j}\right)_{1, N},\left(a_{j}, \alpha_{j}^{\prime}\right)_{N+1, P}}\right]\right\} \\
= & y^{-h} \sum_{k_{1}=0}^{\left[n_{1} / m_{1}\right]} \frac{\left(-n_{1}\right)_{m_{1}, k_{1}}}{k_{1} !} A^{\prime \xi} A_{n_{1}, k_{1}} \sum_{G=0}^{\infty} \sum_{g=1}^{M_{1}} \frac{(-1)^{G}}{G ! F_{g}} \phi_{2}\left(\eta_{G}\right) \tau^{\eta_{G}}\left(\frac{x}{y}\right)^{\sigma \eta_{G}+\xi k_{1}} \\
& \times \bar{H}_{P+1, Q+1}^{M, N+1}\left[\left.w\left(\frac{x}{y}\right)^{\lambda}\right|_{\left(b_{j}, \beta_{j}^{\prime}\right)_{1, M},\left(b_{j}, \beta_{j}^{\prime}: B_{j}\right)_{M+1, Q},\left(1-\mu-\sigma \eta_{G}-\xi k_{1}, \lambda ; 1\right)} ^{\left(1-h-\sigma \eta_{G}-\xi k_{1}, \lambda ; 1\right),\left(a_{j}, \alpha_{j}^{\prime}: A_{j}\right)_{1, N},\left(a_{j}, \alpha_{j}^{\prime}\right)_{N+1, P}}\right] .
\end{aligned}
$$

(3) The result obtained by Srivastava, H. M. and Raina, R. K. (1992) follow as special cases of our result on assigning certain values to parameter in the functions involved.

(4) On taking $A_{j}(j=1, \ldots, N)=B_{j}(j=M+1, \ldots, Q)=1$ and $\sigma \rightarrow 0$ in (2.1) and (2.3) the results reduce to known result recently derived by Goyal, S. P. and Mukherjee, Rohit (2004) with $t=0$.

(5) Letting $A_{j}(j=1, \ldots, N)=B_{j}(j=M+1, \ldots, Q)=1$ and $\sigma \rightarrow 0$ in (2.6) and $n=q=k=B=0$ and $\ell=r=-1$ and $A=1$, the result reduces to a known result derived by Chaurasia, V. B. L. and Patni, Rinku (2001) with $n=0$.

(6) Letting $A_{j}(j=1, \ldots, N)=B_{j}(j=M+1, \ldots, Q)=1$ and $\sigma \rightarrow 0$ in (2.14) and $n=q=k=B=0$, $\ell=r=-1$ and $A=1$, the result reduces to a known result derived by Chaurasia, V. B. L. and Shekhawat, Ashok Singh (2005) with $n=0$

(7) Taking $A_{j}(j=1, \ldots, N)=B_{j}(j=M+1, \ldots, Q)=1$ and $\sigma \rightarrow 0$ in (2.6), we find a known result of Goyal, S. P. and Mukherjee, Rohit (2004) with $t=0$.

\section{Acknowledgement}

The authors are grateful to Professor H. M. Srivastava, University of Victoria, Canada for his kind help and valuable suggestions in the preparation of this paper.

\section{References}

[1] S. K. Chatterjea, Some operational formulas connected with a function defined by a generalized Rodrigues formula, Acta Math. Acad. Hungar 17(1966), 379.

[2] S. K. Chatterjea, Quelques fonctions génératrics des polynomes d'Hermite, du point del'vue de algebre de Lie, C. R. Acad. Sci. Paris, Ser. A-B 268(1969), A 600.

[3] V. B. L. Chaurasia and Ashok Singh Shekhawat, Fredholm type integral equations and certain polynomials, Kyungpook Math. J. 45(2005), 471-481.

[4] V. B. L. Chaurasia and R. Patni, Integral equations of Fredholm type with special functions, Indian J. Pure Appl. Math. 32(2001), 747.

[5] A. Erdélyi et al., Tables of Integral Transforms, McGraw-Hill, New York, 1954. 
[6] C. Fox, The G and H functions as symmetrical Fourier kernels, Trans. Amer. Math. Soc. 98(1961), 395-429.

[7] H. W. Gould and A. T. Hopper, Operational formulas connected with two generalizations of Hermite polynomials, Duke Math. J. 29(1962), 51.

[8] S. P. Goyal and Rohit Mukherjee, A class of convolution integral equations involving product of generalized polynomial set, general class of polynomials and Fox's $H$-function, Bull. Cal. Math. Soc. 96(2004), 265-272.

[9] A. A. Inayat-Hussain, New properties of hypergeometrical series derivable from Feynman integrals, I. Transformation and reductions formula, J. Phys. A. Math. Gen. 20(1987), 4109-4117.

[10] A. A. Inayat-Hussain, New properties of hypergeometrical series derivable from Feynman integrals, II. A generalization of the H-function, J. Phys. A. Math. Gen. 20(1987), 4119-4128.

[11] H. L. Krall and O. Frink, A new class of orthogonal polynomials; the Bessel polynomials, Trans. Amer. Math. Soc. 65(1949), 100.

[12] K. S. Miller, The Weyl fractional calculus in 'Fractional Calculus and its Applications' (B. Ross, ed), Springer Verlag, Berlin, 80, 1975.

[13] K. S. Miller and B. Ross, An introduction to the Fractional Calculus and Fractional Differential Equations, John Willey \& Sons, New York, 1993.

[14] P. Skibiñski, Some expansion theorems for the H-function, Ann. Polon. Math. 23(1970), 125.

[15] H. M. Srivastava, A contour integral involving Fox's H-functions, Indian J. Math. 14(1972).

[16] H. M. Srivastava and R. K. Raina, On certain methods of solving a class of integral equations of Fredholm type, The Journal of the Australian Mathematical Society Ser. A 52(1992), 1-10.

[17] H. M. Srivastava and J. P. Singhal, A class of polynomials defined by generalized Rodrigues formula, Ann. Mat. Pure Appl. 90(1971), 75.

Department of Mathematics, University of Rajasthan, Jaipur-302004, Rajasthan, India.

Department of Mathematics, University of Rajasthan, Jaipur-302004, Rajasthan, India. 